\title{
Ischemic postconditioning attenuates inflammation in rats following renal ischemia and reperfusion injury
}

\author{
HUI CHEN ${ }^{1}$, LEI WANG ${ }^{1}$, BIAN-ZHI XING ${ }^{2}$, XIU-HENG LIU ${ }^{1}$, \\ ZHI-YUAN CHEN ${ }^{1}$, XIAO-DONG WENG ${ }^{1}$, TAO QIU ${ }^{1}$ and LIN LIU ${ }^{1}$ \\ Departments of ${ }^{1}$ Urology and ${ }^{2}$ Radiology, Renmin Hospital of Wuhan University, Wuhan, Hubei 430060, P.R. China
}

Received April 27, 2014; Accepted November 13, 2014

DOI: 10.3892/etm.2015.2514

\begin{abstract}
Ischemic postconditioning (IPoC) involves a series of brief rapid intermittent ischemic episodes applied at the onset of reperfusion in the previously ischemic tissue or organ. Previous studies have demonstrated that IPoC attenuates tissue damage induced by ischemia and reperfusion (I/R) injury. The aim of the present study was to investigate whether IPoC has a beneficial effect on inflammation in a rat model of renal $\mathrm{I} / \mathrm{R}$ injury. Wistar rats were subjected to $45 \mathrm{~min}$ of ischemia followed by 24,72 or $120 \mathrm{~h}$ of reperfusion (I/R group). In the IPoC group, rats subjected to I/R were treated with six cycles of $10 \mathrm{sec}$ reperfusion followed by a 10 -sec ischemic episode. Blood samples were collected for the determination of blood urea nitrogen (BUN) and creatinine $(\mathrm{Cr})$ levels. Furthermore, histological examination and immunohistochemical staining for the localization of nuclear factor- $\kappa \mathrm{B}(\mathrm{NF}-\kappa \mathrm{B})$ were performed. In addition, quantitative polymerase chain reaction (qPCR) analysis was used to determine the expression levels of intercellular adhesion molecule-1 (ICAM-1), interleukin-6 (IL-6) and tumor necrosis factor- $\alpha$ (TNF- $\alpha$ ), while western blot analysis was used to detect the protein expression levels of NF- $\kappa B$. The results indicated that the BUN and $\mathrm{Cr}$ levels increased significantly in the I/R group, while the IPoC rats showed evidently reduced renal damage. Immunohistochemical analysis revealed that the expression levels of $\mathrm{NF}-\kappa \mathrm{B}$ were decreased by IPoC. In addition, the qPCR results revealed that IPoC
\end{abstract}

Correspondence to: Dr Lei Wang, Department of Urology, Renmin Hospital of Wuhan University, 238 Jiefang Road, Wuhan, Hubei 430060, P.R. China

E-mail: drwanglei0045@126.com

Abbreviations: $\mathrm{I} / \mathrm{R}$, ischemia and reperfusion; IPoC, ischemic postconditioning; IPC, ischemic preconditioning; BUN, blood

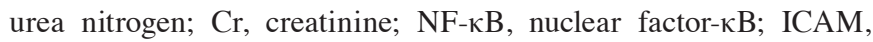
intercellular adhesion molecule; qPCR, quantitative polymerase chain reaction; TNF, tumor necrosis factor; IL, interleukin; PBS, phosphate-buffered saline; TBS, Tris-buffered saline

Key words: ischemic postconditioning, ischemia and reperfusion injury, inflammation, necrosis factor- $\mathrm{\kappa} B$ significantly inhibited the increased mRNA expression levels of ICAM-1, IL-6 and TNF- $\alpha$, induced by I/R injury. Western blot analysis indicated that the expression levels of $\mathrm{NF}-\kappa \mathrm{B}$ were upregulated in the I/R group, while IPoC was shown to inhibit the expression. In conclusion, IPoC was demonstrated to exhibit potent anti-inflammatory properties against renal I/R injury.

\section{Introduction}

Acute kidney injury involves the abrupt loss of renal function, which is strongly associated with increased mortality rates and the subsequent development of chronic kidney disease (1). While acute kidney injury has a number of etiologies, a major cause is ischemia, which can occur during kidney transplantation, renal artery angioplasty, sepsis, partial nephrectomy, accidental or iatrogenic trauma, hydronephrosis, elective urological surgery, aortic bypass surgery and cardiopulmonary bypass, or by the action of vasoconstrictor drugs and certain hypotensive states $(2,3)$.

Previous studies have demonstrated that ischemic preconditioning (IPC) creates resistance against organ ischemia and reperfusion (I/R) injury through 'organ conditioning' $(4,5)$. Although IPC has protective effects and may reduce I/R injury, clinical application is limited and IPC is not suitable for clinical implementation since the onset of an ischemic insult is unpredictable. Ischemic postconditioning (IPoC) involves the application of a series of brief rapid intermittent ischemic episodes at the onset of reperfusion in the previously ischemic tissue or organ (6). IPoC has improved feasibility and operability; thus, is more clinically applicable and attracts greater attention compared with IPC.

Recent studies have indicated that IPoC significantly reduces the inflammatory response in cerebral and lung I/R injury $(7,8)$. An excessive inflammatory reaction frequently resides in the tissue subjected to the I/R injury and leads to damage (9). Therefore, reducing the inflammatory injury is regarded as a major technique for the attenuation of I/R injury. In a previous study, IPoC was demonstrated to attenuate renal damage following I/R injury (10). However, the ability of IPoC to reduce the inflammatory response induced by renal I/R injury has not yet been investigated. Therefore, the aim of the present study was to determine whether IPoC inhibits inflammation following renal I/R injury. 


\section{Materials and methods}

Animal model of $I / R$. In total, 56 adult healthy male Wistar rats (specific-pathogen-free grade; weight, 210-250 g) were supplied by the Animal Experimental Center of the Medical College of Wuhan University (Wuhan, China). The study was approved by the Committee of the Animal Experimental Center of Wuhan University, and the procedures were conducted according to the routine animal-care guidelines. All the experimental procedures complied with the Guidelines for the Care and Use of Laboratory Animals. Briefly, the rats were anesthetized using pentobarbital $(45 \mathrm{mg} / \mathrm{kg})$ and placed on a homeothermic table in order to maintain a core body temperature of $37^{\circ} \mathrm{C}$. A midline laparotomy incision was made and a right nephrectomy was performed. Next, the left kidney was subjected to $45 \mathrm{~min}$ of ischemia, followed by reperfusion.

The animals were randomly divided into three groups of eight rats each, including the sham-operated (sham), I/R and IPoC groups. In the I/R and IPoC groups, the reperfusion period was $24 \mathrm{~h}, 72 \mathrm{~h}$ and $120 \mathrm{~h}$ and the number of rats in each group were as follows: Sham (8 rats), I/R $24 \mathrm{~h}$ (8 rats) ,I/R 72 h (8 rats), I/R 120 h (8 rats), IPoC 24 h (8 rats), IPoC $72 \mathrm{~h}$ (8 rats) and IPoC $120 \mathrm{~h}$ (8 rats). In the sham group, the rats were subjected to a resection of the right kidney. In the I/R group, after the right kidney was removed, the left kidney vessels were subjected to ischemia for $45 \mathrm{~min}$, followed by reperfusion. In the IPoC group, the rats were subjected to 45 min of ischemia, after which the left kidney was immediately subjected to six cycles of reperfusion for $10 \mathrm{sec}$ and a 10 -sec ischemic episode, followed by reperfusion. All the ischemic kidneys were harvested following a reperfusion period of 24,72 or $120 \mathrm{~h}$.

Preservation of the kidneys. The left kidney was removed under fully maintained anesthesia. Following removal, the kidney was fixed in $10 \%$ phosphate-buffered formalin or immediately frozen, and stored at $-80^{\circ} \mathrm{C}$ for the following experiments.

Serum assays. At $24 \mathrm{~h}$ following the initiation of I/R injury, in every group, 1-ml blood samples were collected and analyzed according to directions of the Creatinine and Urea Assay kits (Nanjing Jiancheng Chemical Industrial Co., Ltd, Nanjing, China). The absorbance was measured using a spectrophotometer (Shimadzu UV-1700; Shimadzu Corporation, Kyoto, Japan) and the concentrations of blood urea nitrogen (BUN) and creatinine $(\mathrm{Cr})$ were calculated.

Histological examinations. The kidneys were fixed in $10 \%$ phosphate-buffered formalin, embedded in paraffin and cut into $4-\mu \mathrm{m}$ sections. The sections were deparaffinized and hydrated gradually, followed by staining with hematoxylin and eosin. Morphological assessments were performed by an experienced renal pathologist who was unaware of the assigned treatments. An established grading scale of $0-4$, outlined by Jablonski et al (11), was used in the histopathological assessment of $\mathrm{I} / \mathrm{R}$-induced damage.

Immunohistochemistry. Immunohistochemical staining was used to analyze the expression of nuclear factor $-\kappa B(N F-\kappa B)$.
Briefly, the 4- $\mu \mathrm{m}$ sections were deparaffinized, and endogenous peroxidase activity was blocked with $3 \%$ hydrogen peroxide for $10 \mathrm{~min}$ at $37^{\circ} \mathrm{C}$. Next, the sections were treated with $10 \%$ normal goat serum in Tris-buffered saline (TBS) for $30 \mathrm{~min}$ at $37^{\circ} \mathrm{C}$. Subsequently, the samples were incubated overnight at $4{ }^{\circ} \mathrm{C}$ with a polyclonal rabbit anti-NF- $\kappa \mathrm{B}$ antibody (no. ab16502; 1:1,000; Abcam, Cambridge, UK). After washing three times with phosphate-buffered saline (PBS), the sections were incubated with a goat anti-rabbit secondary antibody (1:2,000; ZSGB-BIO Corporation, Beijing, China) for $30 \mathrm{~min}$ at room temperature, followed by the addition of the color reagent, 3,3'-diaminobenzidine. The aforementioned experiments were routinely performed in the negative control group although PBS was used instead of incubation with the primary antibody.

Quantitative polymerase chain reaction ( $q P C R)$. Total RNA was isolated using TRIzol reagent (Invitrogen Life Technologies, Carlsbad, CA, USA), and the RNA concentration was determined using a spectrophotometer (Shimadzu UV-1700; Shimadzu Corporation). Single-stranded cDNA was synthesized with a RevertAid First Strand cDNA synthesis kit (Takara Bio, Inc., Kyoto, Japan), according to the manufacturer's instructions. Reverse transcription PCR was performed using the SYBR ${ }^{\circledast}$ Green mix kit (Applied Biosystems Life Technologies, Foster City, CA, USA). The primers used were as follows: Tumor necrosis factor- $\alpha$ (TNF- $\alpha$ ) forward, 5'-GCCACCACGCTCTTCTGTC-3' and reverse, 5'-GCTACGGGCTTGTCACTCG-3' (GenBank accession no. NM_012675.3); intercellular adhesion molecule-1 (ICAM-1) forward, 5'-GGGATGGTGAAGTCTGTCAA-3' and reverse, 5'-GGCGGTAATAGGTGTAAATGG-3' (GenBank accession no. NM_012967); and interleukin-6 (IL-6) forward, 5'-TTGCCTTCTTGGGACTGATGT-3' and reverse, 5'-TACTGGTCTGTTGTGGGTGGT-3' (GenBank accession no. NM_012589.1). $\beta$-actin was used as a housekeeping gene and the data were presented as the ratio of gene expression against that of $\beta$-actin. The $\beta$-actin sense primer used was 5'-TGCTATGTTGCCCTAGACTTCG-3' and the antisense primer was 5'-GTTGGCATAGAGGTCTTTACGG-3' (GenBank accession no. NM_031144). The initial activation was at $95^{\circ} \mathrm{C}$ for $15 \mathrm{sec}$, followed by $58^{\circ} \mathrm{C}$ for $20 \mathrm{sec}$ and $72^{\circ} \mathrm{C}$ for $20 \mathrm{sec}$ for 40 cycles. SLAN ${ }^{\circledR}-96$ s Real-Time PCR system (Shanghai Hongshi Medical Technology Co., Ltd, Shanghai, China) was used to carry out the analysis. There were three samples per assay.

Western blot analysis. Total proteins were extracted and quantified using a bicinchoninic acid assay. Next, equivalent protein samples (40 $\mu \mathrm{g} /$ lane) were separated using $10 \%$ SDS-PAGE and transferred to nitrocellulose membranes. The membranes were blocked with $5 \%$ non-fat milk in TBS/Tween-20, and incubated with polyclonal rabbit anti-NF- $\mathrm{B}$ primary antibodies against NF- $\mathrm{\kappa B}$ (no. ab16502; 1:1,000; Abcam). Following two washes with PBS, the membranes were incubated with a goat anti-rabbit secondary antibody conjugated to horseradish peroxidase $(1: 2,000$; ZSGB-BIO Corporation). Specific bands were visualized using an enhanced chemiluminescence detection kit (Pierce Biotechnology, Inc., Rockford, IL, USA), and the 
A

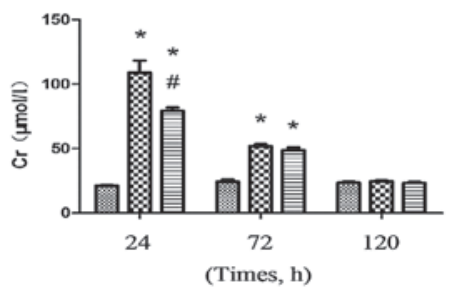

B

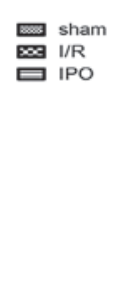

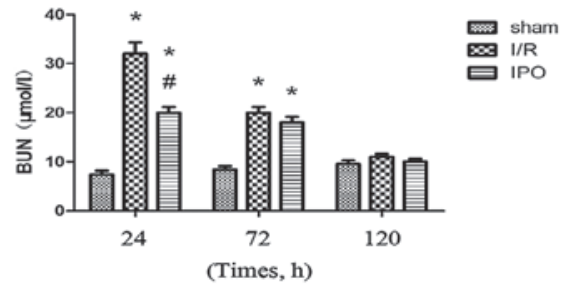

$\mathrm{C}$

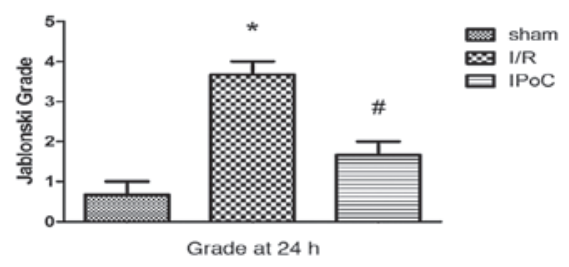

Figure 1. Effect of IPoC on the (A) serum Cr and (B) BUN concentrations, and (C) Jablonski grading scale scores, after $24 \mathrm{~h}$. The data are presented as the mean \pm standard error of mean. "P $<0.05$, vs. sham; ${ }^{\#} \mathrm{P}<0.05$, vs. I/R at $24 \mathrm{~h} . \mathrm{Cr}$, creatinine; BUN, blood urea nitrogen; sham, sham-operated; I/R, ischemia and reperfusion; IPoC, ischemic postconditioning.
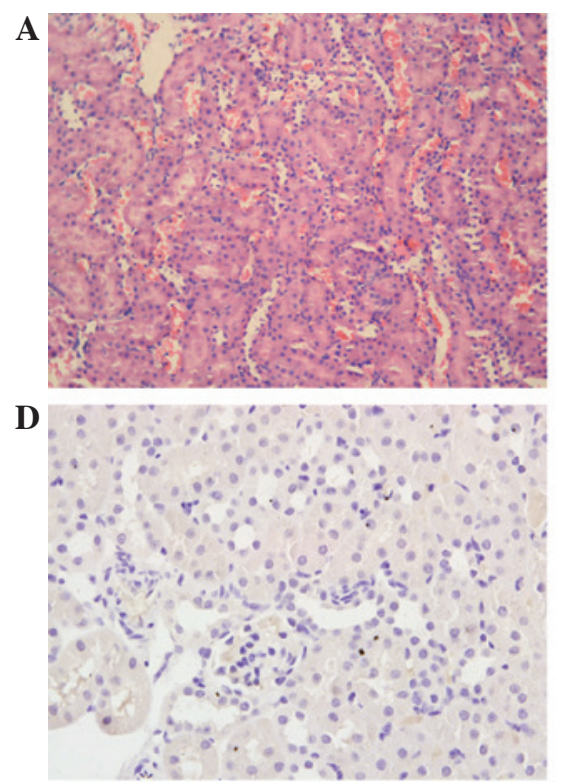

$\mathbf{B}$

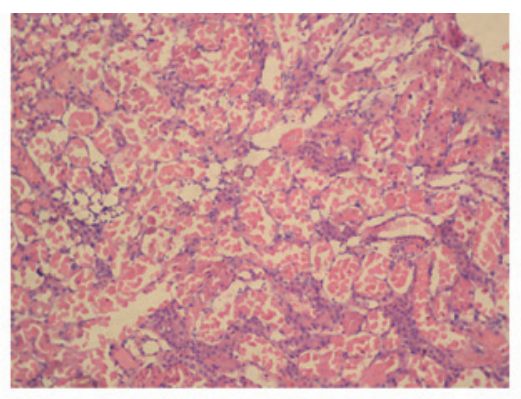

$\mathbf{E}$

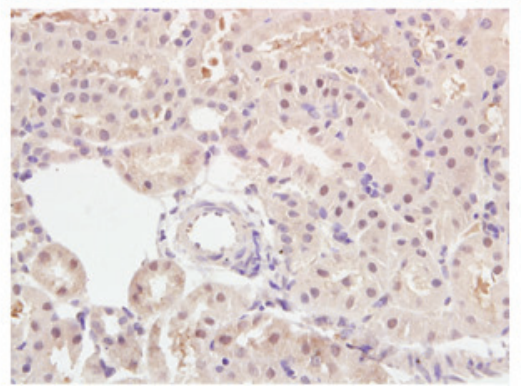

C

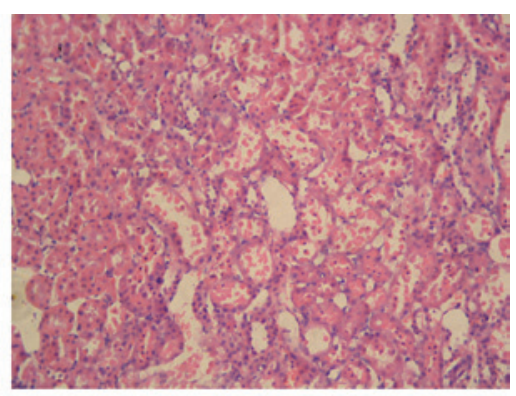

F

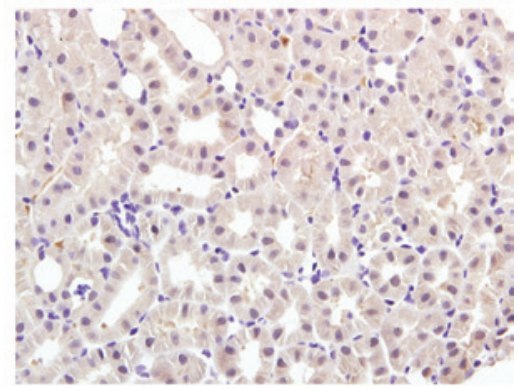

Figure 2. Histological features were evaluated using hematoxylin and eosin (H\&E) staining (magnification, x200), while immunohistochemical staining (magnification, $\mathrm{x} 400$ ) was used to investigate the expression of nuclear factor- $\mathrm{\kappa B}(\mathrm{NF}-\mathrm{\kappa B})$. Representative kidney sections stained with $\mathrm{H} \& \mathrm{E}$ at the end of the 24-h reperfusion period are shown for the (A) sham, (B) I/R and (C) IPoC groups. The expression of NF- $\mathrm{kB}$ in the kidneys at the end of the 24-h reperfusion period is shown for the (D) sham, (E) I/R and (F) IPoC groups. Sham, sham-operated; I/R, ischemia and reperfusion; IPoC, ischemic postconditioning.

optical densities were detected using Quantity One software (Bio-Rad Laboratories, Inc., Hercules, CA, USA).

Statistical analysis. Data are presented as the mean \pm standard error of mean. The mean values of the groups were compared using one-way analysis of variance, followed by the Student-Newman-Keuls test. All statistical analyses were performed with the SPSS statistical package (SPSS 13.0; SPSS, Inc., Chicago, IL, USA). $\mathrm{P}<0.05$ was considered to indicate a statistically significant difference.

\section{Results}

Renal function. Renal function was found to be different at each of the three reperfusion time points (24, 72 and $120 \mathrm{~h}$ ). The renal functional parameters of the rats were significantly different at 24 and $72 \mathrm{~h}$ following I/R injury. Rats that were subjected to $\mathrm{I} / \mathrm{R}$ injury showed a significant increase in the levels of BUN and $\mathrm{Cr}$ compared with the sham-operated rats at 24 and $72 \mathrm{~h}$ following I/R injury. In addition, the renal function after I/R injury was significantly improved following IPoC treatment (Fig. 1).

Histopathology. Compared with the sham group, the I/R group rats suffered from significant tubular necrosis, medullary hemorrhage and congestion. However, IPoC administration reduced these severe renal damages (Fig. 2A-C). Quantitative analysis revealed a markedly decreased Jablonski score in the IPoC group rats compared with the I/R group (Fig. 1C).

Immunohistochemistry. $\mathrm{NF}-\kappa \mathrm{B}$ expression was detected using an immunohistochemical technique. Staining revealed that the $\mathrm{NF}-\kappa \mathrm{B}$ expression level was low in the sham group. However, the renal tissues of the I/R group exhibited a strong positive expression for $\mathrm{NF}-\kappa \mathrm{B}$. Compared with the I/R group, the expression levels of $\mathrm{NF}-\kappa \mathrm{B}$ were decreased in the IPoC group (Fig. 2D-F).

PCR analysis. The mRNA expression levels of ICAM-1, IL-6 and TNF- $\alpha$ were calculated relative to $\beta$-actin. The expression 
A

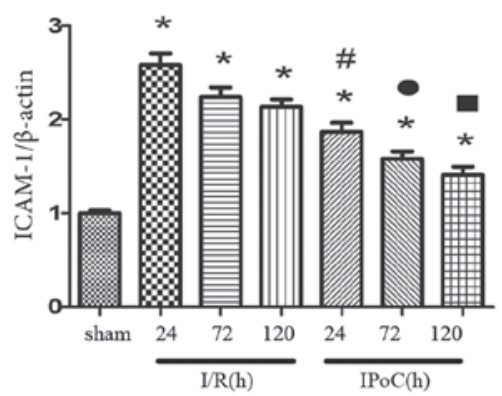

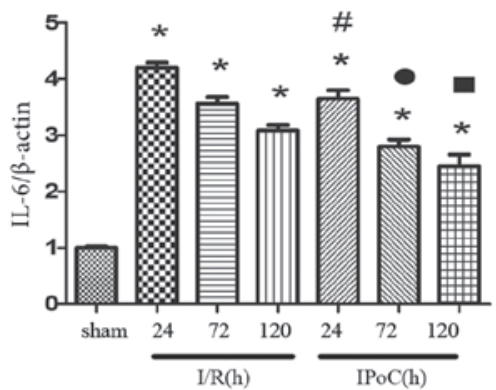

C

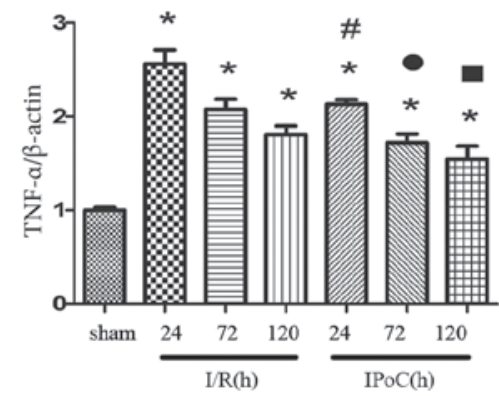

Figure 3. Effect of IPoC on the mRNA expression levels of (A) ICAM-1, (B) IL-6 and (C) TNF- $\alpha$ in the kidney after 45 min of ischemia, followed by 24,72 and $120 \mathrm{~h}$ of reperfusion. The mRNA expression levels were standardized against $\beta$-actin. The data are presented as the mean \pm standard error of mean. ${ }^{*} \mathrm{P}<0.05$, vs. sham; ${ }^{*} \mathrm{P}<0.05$, vs. I/R at $24 \mathrm{~h} ;{ }^{\circ} \mathrm{P}<0.05$, vs. I/R at $72 \mathrm{~h}$; $\mathrm{P}<0.05$, vs. I/R at $120 \mathrm{~h}$. TNF- $\alpha$, tumor necrosis factor- $\alpha$; IL-6, interleukin-6; ICAM-1, intercellular adhesion molecule-1; sham, sham-operated; I/R, ischemia and reperfusion; IPoC, ischemic postconditioning.

A

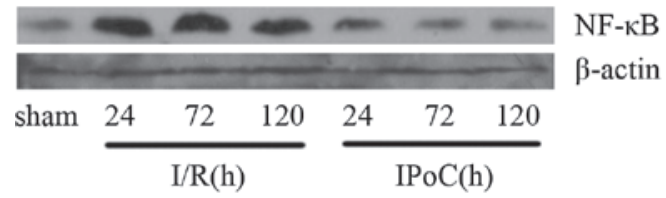

B

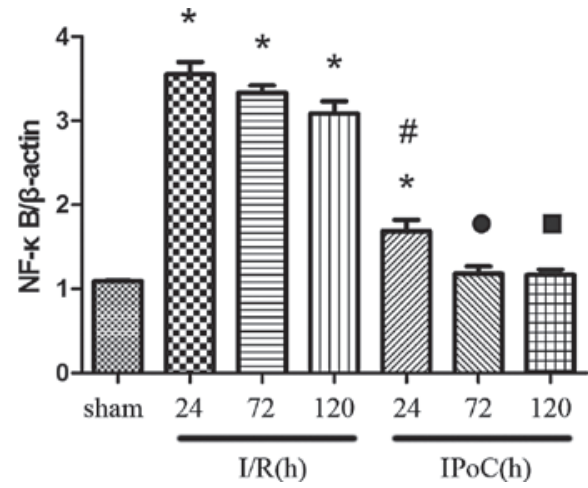

Figure 4. (A) Representative western blot and (B) quantitative analyses revealing the effects of IPoC on the expression of NF- $\kappa \mathrm{B}$ in the kidney after 45 min of ischemia, followed by 24,72 and $120 \mathrm{~h}$ of reperfusion. $\beta$-actin was used to ensure an equal amount of protein was loaded in each lane. The data are presented as the mean \pm standard error of mean. ${ }^{*} \mathrm{P}<0.05$, vs. sham; ${ }^{\#} \mathrm{P}<0.05$, vs. I/R at $24 \mathrm{~h} ;{ }^{*} \mathrm{P}<0.05$, vs. I/R at $72 \mathrm{~h}$; ${ }^{-P}<0.05$, vs. I/R at $120 \mathrm{~h}$. NF- $\kappa \mathrm{B}$, nuclear factor- $\kappa \mathrm{B}$; sham, sham-operated; $\mathrm{I} / \mathrm{R}$, ischemia and reperfusion; IPoC, ischemic postconditioning.

levels were found to vary following 24,72 and $120 \mathrm{~h}$ of reperfusion, and were significantly higher in the I/R group compared with the sham group. However, IPoC was shown to significantly reduce the mRNA expression levels of ICAM-1, IL-6 and TNF- $\alpha$ following I/R (Fig. 3).

Western blot analysis. Compared with the sham group, the expression levels of NF- $\mathrm{KB}$ were upregulated in the I/R group, and gradually decreased with time. However, IPoC was found to attenuate the NF- $\kappa B$ expression levels induced by $I / R$, as shown in Fig. 4.

\section{Discussion}

In recent years, $\mathrm{I} / \mathrm{R}$ injury has elicited an increasing interest due to the impact on organs, such as the kidney, liver and heart. In addition, research into the protective effects of "organ conditioning' against I/R injury has received increasing interest. IPC has been demonstrated to protect organs against the tissue damage induced by $I / R$, and the underlying mechanisms were shown to involve a complex set of signaling transduction pathways (12). However, clinical application of IPC is often restricted since the onset of ischemic injury is difficult to predict (13). A more clinically suitable approach is IPoC, performed at the onset of reperfusion. IPoC was first reported by Zhao et al (6) as an effective strategy against cardiac I/R injury. The protective effects of IPoC have also been demonstrated in several animal models of non-cardiac I/R injury (14). Our previous study revealed that IPoC attenuated oxidative stress and protected rats against renal I/R injury; however, a protective mechanism directly involving the kidney was not elucidated. Our study supported and further demonstrated the aforementioned findings, revealing that IPoC was able to reduce the expression levels of BUN and $\mathrm{Cr}$ and improve renal morphology following I/R injury (10). In addition, IPoC was shown to inhibit inflammation following renal I/R injury in rats, for the first time.

Inflammation is a key factor in the occurrence and development of ischemic damage. The activation of monocytes and macrophages contributes to the synthesis and release of a variety of proinflammatory cytokines following $I / R$ injury (15). Within hours after an ischemic episode, a large number of proinflammatory mediators are released, leading to the development of tissue damage. Among the pathological processes involved in I/R injury, TNF- $\alpha$ plays a key role in the development and maintenance of an inflammatory response. The infiltration of leukocytes into the kidney, assisted by TNF- $\alpha$, may aggravate the ischemic injury (16). In addition, ICAM-1, an adhesion molecule, facilitates leukocyte infiltration and adhesion, aggravating the injuries caused by I/R. A 
previous study demonstrated that the functional inhibition of TNF- $\alpha$, which was associated with the decreased expression of ICAM-1, was able to reduce the extent of I/R injury (17). IL-6 is a pleiotropic cytokine involved in the regulation of immune and inflammatory responses. In the present study, the increased expression levels of IL- 6 , TNF- $\alpha$ and ICAM-1, which are markers of inflammation, were reduced by IPoC. Thus, IPoC was found to reduce the inflammatory responses following renal I/R injury. The mRNA expression levels of TNF- $\alpha$, IL-6 and ICAM-1 in the I/R group increased significantly in response to the $\mathrm{I} / \mathrm{R}$-induced renal damage, peaking after $24 \mathrm{~h}$ of reperfusion, followed by a gradual decrease. However, IPoC was shown to significantly protect the renal tissue from I/R injury, since the increased expression levels of the inflammatory markers were reduced markedly in the IPoC group when compared with the I/R group (Fig. 3). Therefore, the results demonstrated that IPoC reduces the inflammatory response following renal I/R injury.

$\mathrm{NF}-\kappa \mathrm{B}$, an important nuclear transcription factor, regulates the expression of a large number of genes that play a key role in the regulation of apoptosis, inflammation, viral replication and tumorigenesis (18). Normally, an inactive form of $\mathrm{NF}-\kappa \mathrm{B}$ is sequestered in the cytoplasm bound to I $\kappa \mathrm{B}$ proteins, which regulates its activity (19). Numerous stimuli, including I/R injury, can activate $\mathrm{NF}-\kappa \mathrm{B}$ signaling through the degradation of $\mathrm{I} \kappa \mathrm{B}$ and the release of the NF- $\kappa \mathrm{B}$ p65-p50 dimer. The dimer translocates to the nucleus, binds to the $\kappa \mathrm{B}$ binding sites of DNA and regulates the transcriptional activation of target genes (20). NF- $\mathrm{BB}$ is crucial in the regulation of genes encoding proinflammatory cytokines, such as IL-6, TNF- $\alpha$ and ICAM-1. A previous study demonstrated that $\mathrm{NF}-\kappa \mathrm{B}$ may be a vital regulator of inflammation following kidney damage, with inflammatory mechanisms shown to be closely associated with increased expression levels of NF- $\mathrm{B}$ (21). In the present study, the expression of $\mathrm{NF}-\kappa \mathrm{B}$ was investigated following 24, 72 and $120 \mathrm{~h}$ of reperfusion in the I/R and IPoC groups. The results indicated that the expression levels of NF- $\kappa \mathrm{B}$ were upregulated in the I/R group after $24 \mathrm{~h}$ of reperfusion and then gradually decreased. By contrast, IPoC was found to significantly attenuate the expression levels induced by I/R injury, which was consistent with the changes observed in renal function.

However, a number of limitations exist in the present study. A recent clinical study demonstrated that IPoC did not reduce the delayed graft function or improve renal function following kidney transplantation, although IPoC application was found to be feasible and safe (22). A possible explanation for the conflicting results may be that healthy young animals are used in the majority of animal experiments, while in the aforementioned clinical study, the transplant donors were older and suffered from a number of comorbidities. Therefore, future studies should investigate aged or diseased rats. Furthermore, in vitro studies are required to confirm the results, since an inherent interconnection of the effects of IPoC treatment on tissue salvage and protein signals was observed. In addition, only a short-term period of survival was assessed in the present study, while a previous study demonstrated that IPoC protected rats against I/R damage after 12 weeks and had beneficial effects on renal fibrosis (23). The anti-inflammatory properties of IPoC may possibly lead to the long-term protection of renal fibrosis; therefore, the long-term consequences of IPoC shoulwd be investigated in a further study. Furthermore, only six cycles of reperfusion for $10 \mathrm{sec}$ followed by $10 \mathrm{sec}$ of ischemia were applied for IPoC. Thus, the current study did not reveal whether IPoC plays an 'on-off' or 'dose-dependent' role. In the case that IPoC is 'dose-dependent', the ischemic episode period of $10 \mathrm{sec}$ may not afford the maximal protective effect against renal I/R injury. Thus, the optimal interval length and number of cycles require further investigation.

In conclusion, IPoC was demonstrated to protect rats against inflammation following renal $\mathrm{I} / \mathrm{R}$ injury, and the underlying mechanism of IPoC was found to be associated with the decreased expression of $\mathrm{NF}-\kappa \mathrm{B}$. Therefore, inhibiting the activation of $\mathrm{NF}-\kappa \mathrm{B}$ may develop smaller impairments following renal I/R injury.

\section{Acknowledgements}

The study was supported by a grant from the National Natural Science Foundation of China (no. 30901494).

\section{References}

1. Kam Tao Li P, Burdmann EA and Mehta RL; World Kidney Day Steering Committee 2013: Acute kidney injury: Global health alert. J Nephropathol 2: 90-97, 2013.

2. Yun Y, Duan WG, Chen P, Wu HX, Shen ZQ, Qian ZY and Wang DH: Ischemic postconditioning modified renal oxidative stress and lipid peroxidation caused by ischemic reperfusion injury in rats. Transplant Proc 41: 3597-3602, 2009.

3. Barri YM, Sanchez EQ, Jennings LW, Melton LB, Hays S, Levy MF and Klintmalm GB: Acute kidney injury following liver transplantation: definition and outcome. Liver Transpl 15: 475-483, 2009.

4. Ma ZF, Chen W, Cao CC and Chen X: Ischemic preconditioning attenuates brain injury induced by ischemia/reperfusion during moderate hypothermia low-flow procedures. Int J Neurosci: Jan 24, 2014 (Epub ahead of print).

5. Ma J, Qiao Z and Xu B: Effects of ischemic preconditioning on myocardium Caspase-3, SOCS-1, SOCS-3, TNF- $\alpha$ and IL-6 mRNA expression levels in myocardium IR rats. Mol Biol Rep 40: 5741-5748, 2013.

6. Zhao ZQ, Corvera JS, Halkos ME, Kerendi F, Wang NP, Guyton RA and Vinten-Johansen J: Inhibition of myocardial injury by ischemic postconditioning during reperfusion: comparison with ischemic preconditioning. Am J Physiol Heart Circ Physiol 285: H579-H588, 2003.

7. Kong Y, Rogers MR and Qin X: Effective neuroprotection by ischemic postconditioning is associated with a decreased expression of RGMa and inflammation mediators in ischemic rats. Neurochem Res 38: 815-825, 2013

8. Wu H, Lei S, Yuan J, Liu X, Zhang D, Gu X, Zhang L and Xia Z: Ischemic postconditioning downregulates Egr-1 expression and attenuates postischemic pulmonary inflammatory cytokine release and tissue injury in rats. J Surg Res 181: 204-212, 2013.

9. Chen H, Xing B, Liu X, Zhan B, Zhou J, Zhu H and Chen Z: Ozone oxidative preconditioning inhibits inflammation and apoptosis in a rat model of renal ischemia/reperfusion injury. Eur J Pharmacol 581: 306-314, 2008.

10. Chen H, Xing B, Liu X, Zhan B, Zhou J, Zhu H and Chen Z: Ischemic postconditioning inhibits apoptosis after renal ischemia/reperfusion injury in rat. Transpl Int 21: 364-371, 2008.

11. Jablonski P, Howden BO, Rae DA, Birrell CS, Marshall VC and Tange J: An experimental model for assessment of renal recovery from warm ischemia. Transplantation 35: 198-204, 1983.

12. Granfeldt A, Lefer DJ and Vinten-Johansen J: Protective ischaemia in patients: preconditioning and postconditioning. Cardiovasc Res 83: 234-246, 2009.

13. Venugopal V, Ludman A, Yellon DM and Hausenloy DJ: 'Conditioning' the heart during surgery. Eur J Cardiothorac Surg 35: 977-987, 2009. 
14. Zhao ZQ: Postconditioning in reperfusion injury: a status report. Cardiovasc Drugs Ther 24: 265-279, 2010.

15. Wang JQ, Chen X, Zhang C, Tao L, Zhang ZH, Liu XQ, Xu YB, Wang H, Li J and Xu DX: Phenylbutyric acid protects against carbon tetrachloride-induced hepatic fibrogenesis in mice. Toxicol Appl Pharmacol 266: 307-316, 2013

16. Wang Y,GeP and Zhu Y: TLR2 and TLR4 in the brain injury caused by cerebral ischemia and reperfusion. Mediators Inflamm 2013: 124614, 2013.

17. Carden DL and Granger DN: Pathophysiology of ischaemia-reperfusion injury. J Pathol 190: 255-266, 2000.

18. Ma JQ, Liu CM, Qin ZH, Jiang JH and Sun YZ: Ganoderma applanatum terpenes protect mouse liver against benzo $(\alpha)$ pyren-induced oxidative stress and inflammation. Environ Toxicol Pharmacol 31: 460-468, 2011.

19. Li YW, Zhang Y, Zhang L, Li X, Yu JB, Zhang HT, Tan BB, Jiang LH, Wang YX, Liang Y, et al: Protective effect of tea polyphenols on renal ischemia/reperfusion injury via suppressing the activation of TLR4/NF-кB p65 signal pathway. Gene 542: 46-51, 2014.
20. Wong ET and Tergaonkar V: Roles of NF-kappaB in health and disease: mechanisms and therapeutic potential. Clin Sci (Lond) 116: 451-465, 2009.

21. Zhang P, Liu X, Zhu Y, Chen S, Zhou D and Wang Y: Honokiol inhibits the inflammatory reaction during cerebral ischemia reperfusion by suppressing $\mathrm{NF}-\kappa \mathrm{B}$ activation and cytokine production of glial cells. Neurosci Lett 534: 123-127, 2013.

22. van den Akker EK, Hesselink DA, Manintveld OC, et al: Ischemic postconditioning in human DCD kidney transplantation is feasible and appears safe. Transpl Int 27: 226-234, 2014.

23. Weng X, Shen H, Kuang Y, Liu X, Chen Z, Zhu H, Jiang B, Zhu G and Chen $\mathrm{H}$ : Ischemic postconditioning inhibits the renal fibrosis induced by ischemia-reperfusion injury in rats. Urology 80: 484.e1-484.e7, 2012. 